\title{
Progressor: Social Navigation Support through Open Social Student
}

\section{Modeling}

I-Han Hsiao ${ }^{\mathrm{a}}$, Fedor Bakalov ${ }^{\mathrm{b}}$, Peter Brusilovsky ${ }^{\mathrm{a}}$, Birgitta König-Ries ${ }^{\mathrm{b}}$

${ }^{a}$ School of Information Sciences, 135 North Bellefield Avenue, University of Pittsburgh, Pittsburgh, 15260, USA.

$$
\text { phone: +1 (412) 624 9404, fax: + 1 (412) } 6242788
$$

Email:ihh4@pitt.edu; peterb@mail.sis.pitt.edu

${ }^{\mathrm{b}}$ Institute for Computer Science, University of Jena, Ernst-Abbe-Platz 2, 07743 Jena, Germany

phone: +493641 946435

Email: fedor.bakalov@uni-jena.de; birgitta.koenig-ries@uni-jena.de 


\section{Progressor: Social Navigation Support through Open Social Student}

\section{Modeling}

The increased volumes of online learning content produced two problems: how to help students to find the most appropriate resources and how to engage them in using these resources. Personalized and social learning have been suggested as potential ways to address these problems. Our work presented in this paper combines the ideas of personalized and social learning in the context of educational hypermedia. We introduce Progressor, an innovative Web-based tool based on the concepts of social navigation and open student modeling that helps students to find the most relevant resources in a large collection of parameterized self-assessment questions on Java programming. We have evaluated Progressor in a semester-long classroom study, the results of which are presented in this paper. The study confirmed the impact of personalized social navigation support provided by the system in the target context. The interface encouraged students to explore more topics attempting more questions and achieving higher success rates in answering them. A deeper analysis of the social navigation support mechanism revealed that the top students successfully led the way to discovering most relevant resources by creating clear pathways for weaker students.

Keywords: Open student model; Visualization; Personalized E-learning; Social learning; Self-assessment; Personalized information access; Social navigation support

\section{Introduction}

A large volume of educational resources is now available on the Web to support both regular classroom learning and online learning. The abundance of available content has resulted in at least two challenges: how to help students to find the most appropriate resources and how to entice them into using these resources and benefitting from them. These problems encouraged a good deal of interesting research in modern E-Learning. Of special interest in the context of this paper are the personalized and social technologies explored in a number of projects. Personalized learning was suggested as 
an approach to help every learner to find most relevant and useful content given the learner's current state of knowledge and interest (Kay, 2008). Social learning was explored as a potential solution to a range of problems including increasing students' motivation to learn (Barolli et al., 2006; Méndez et al., 2006; Vassileva, 2008; Vassileva and Sun, 2008). The work presented in this paper combines the ideas of personalized and social learning in an educational hypermedia context by offering an open social student modeling interface to guide students to relevant resources in a large collection of parameterized self-assessment questions on Java programming.

This research has been motivated by the success of adaptive navigation support and open student modeling, two very popular approaches in the area of personalized learning. Adaptive navigation support is known to increase learning rates and quality (Brusilovsky, 2007; Kavcic, 2004). Visual approaches for open student modeling have been proven to provide students with an easy-to-grasp and holistic view of their progress (Bull, 2004; Mitrovic and Martin, 2007; Zapata-Rivera and Greer, 2000). However, most of the research on adaptive navigation support and open student modeling focuses on an individual student representation and ignores the social aspects of learning. Our prior experience with social navigation (Farzan and Brusilovsky, 2008) as well as others' work on social learning techniques reviewed below indicated that the ability to see the work and progress of peer students can help to increase both the quality of navigation support and the student's motivation. The goal of research presented in this paper was to explore an innovative social navigation support approach that we call open social student modeling. The idea of this approach is to extend a traditional open student modeling interface with social guidance by allowing the students to view and explore multiple peer models. We implemented several versions of an open social student modeling interface called Progressor and conducted several 
semester-long classroom studies. The studies confirmed the motivational impact of social navigation support provided by the system in the target context. The interface encouraged students to explore more topics and motivated them to do work ahead of the course schedule. A deeper analysis of the social navigation support mechanism revealed that the top students provided implicit social navigation support for the rest of the class and successfully led the way to discovering the most relevant resources by creating clear trails for weaker students. The study results also demonstrated that students were more engaged with the system as they were spending more time in working with selfassessment questions, attempting more questions and achieving higher success rates in answering them.

The rest of the paper is organized as follows. A brief literature review about open user modeling, social navigation support, and social visualization in E-Learning will be presented in the next section. In Section 3, we describe the interface design rationale and underlying features of Progressor. A comprehensive evaluation of Progressor is reported in Section 4. We summarize this work and discuss potential future work in Section 5.

\section{Related Work}

Our work integrates two distinct research directions in personalized and social elearning: open student modeling and social navigation support. A brief review of these research streams is provided below.

\subsection{Open Student Models}

Open student modeling has recently emerged as an important research topic in the field of personalized learning. There are two main streams of work on open student models. One stream focuses on visual presentation student models to support students' self- 
reflection and planning; the other one encourages students to participate in the modeling process by engaging students through the negotiation of or collaboration on construction of the model (Corbett and Anderson, 1995). Visual representations of the student model vary from displaying high-level summaries (such as skill meters) to complex concept maps or Bayesian networks. In (Corbett and Anderson, 1995), the authors describe APTList Tutor, an interface that provides the learner with a skill meter showing the list of learning goals and the progress the learner has made towards those goals. Mabbott and Bull in (Mabbott and Bull, 2004) explored an interface that provides students with four different views of their learner models. These views portray different aspects of the underlying domain knowledge model, namely the hierarchical structure of topics, lecture structure, semantic relationships among the topics, and recommended sequence of learning the topics. The STyLE-OLM interface proposed by Dimitrova in (Dimitrova, 2003) allows students to browse and navigate through their learner models using the visual notation of concept graphs.

A range of benefits have been reported when making the student models available to the learners, such as increasing the learner's awareness of their own developing knowledge; difficulties in the learning process; and students' engagement, motivation, and knowledge reflection (Bull, 2004; Mitrovic and Martin, 2007; ZapataRivera and Greer, 2000). Several researchers (Dimitrova et al., 2001) explore interactive open learner modeling by engaging learners in negotiating with the system during the modeling process. Chen et al. (Chen et al., 2007) investigated active open learner models in order to motivate learners to improve their academic performance. Both individual and group open learner models were studied and demonstrated the increase of reflection and helpful interactions among teammates. In (Bull and Kay, 2007), the authors described a framework to apply open user models in adaptive 
learning environments and provided many in-depth examples. Studies also show that students have a range of preferences for presentation styles when viewing their own knowledge in the open student modeling systems. Students highly value the options of having multiple views and being able to select the one with which they are the most comfortable. Such results are promising for potentially increasing the quality of reflection on their own knowledge (Mabbott and Bull, 2004). In our own work on the QuizGuide system (Hsiao et al., 2010) we integrated open learning models with annotation-based adaptive navigation support and demonstrated that this arrangement can remarkably increase students' motivation to work with non-mandatory educational content.

\subsection{Social Navigation Support}

Social navigation support (Dieberger et al., 2000) belongs to a group of social information access technologies, a stream of research that explores methods for organizing users' previous interactions with an information system (known as explicit and implicit feedback), in order to provide better access to information to the future users of the system (Brusilovsky, 2008). The motivation for this work was that the "footprints" of prior users can help future users to navigate through an information space. The first generation of social navigation support projects explored the simplest and most natural kind of "footprints" - browsing tracks of prior users. This kind of social navigation support is known as traffic-based. An excellent example of trafficbased social navigation is provided by the pioneering systems Juggler (Dieberger, 1997) and Footprints (Wexelblat and Mayes, 1999). This work was very influential in educational hypermedia where navigation support is especially important. Over the last 10 years, a range of traffic-based social navigation approaches has been explored in the 
E-learning context (Brusilovsky et al., 2004; Janssen et al., 2007; Kurhila et al., 2002; Mertens et al., 2006).

Our group also initiated work on educational social navigation with simple traffic based approaches (Brusilovsky et al., 2004); however, we were not satisfied with the quality of traffic-based navigation support. As a result, our most recent projects focused on increasing the reliability of social navigation support by using more reliable "footprints" such as user annotation behavior (Farzan and Brusilovsky, 2008), bookmarking (Farzan and Brusilovsky, 2008), and ratings (Farzan and Brusilovsky, 2006). This paper continues this direction of research by exploring social navigation based on the results of student problem-solving behavior, which provides a much more reliable trace than a simple clickstream.

\section{The Progressor Interface}

Progressor is a combination of social visualization and open learner modeling approaches. It provides students with a holistic and easy-to-grasp view of their progress and permits them to compare it to the progress of other students in the class. The system integrates two earlier projects, namely the QuizJET system (Hsiao et al., 2008) for the authoring and delivery of parameterized questions for the Java programming language and the IntrospectiveViews interface (Bakalov et al., 2010) for visualization of semantic user models. The predecessor of Progressor was introduced in (Hsiao et al., 2011) as Parallel IntrospectiveViews. It offers visualization of student progress with QuizJET questions in an Object-Oriented programming course. All questions are parameterized, i.e., they include a random parameter, which QuizJET instantiates when the question is delivered to a student. As a result, the student can attempt to answer the same question multiple times with different values of the parameter, which helps to reach the mastery level. 


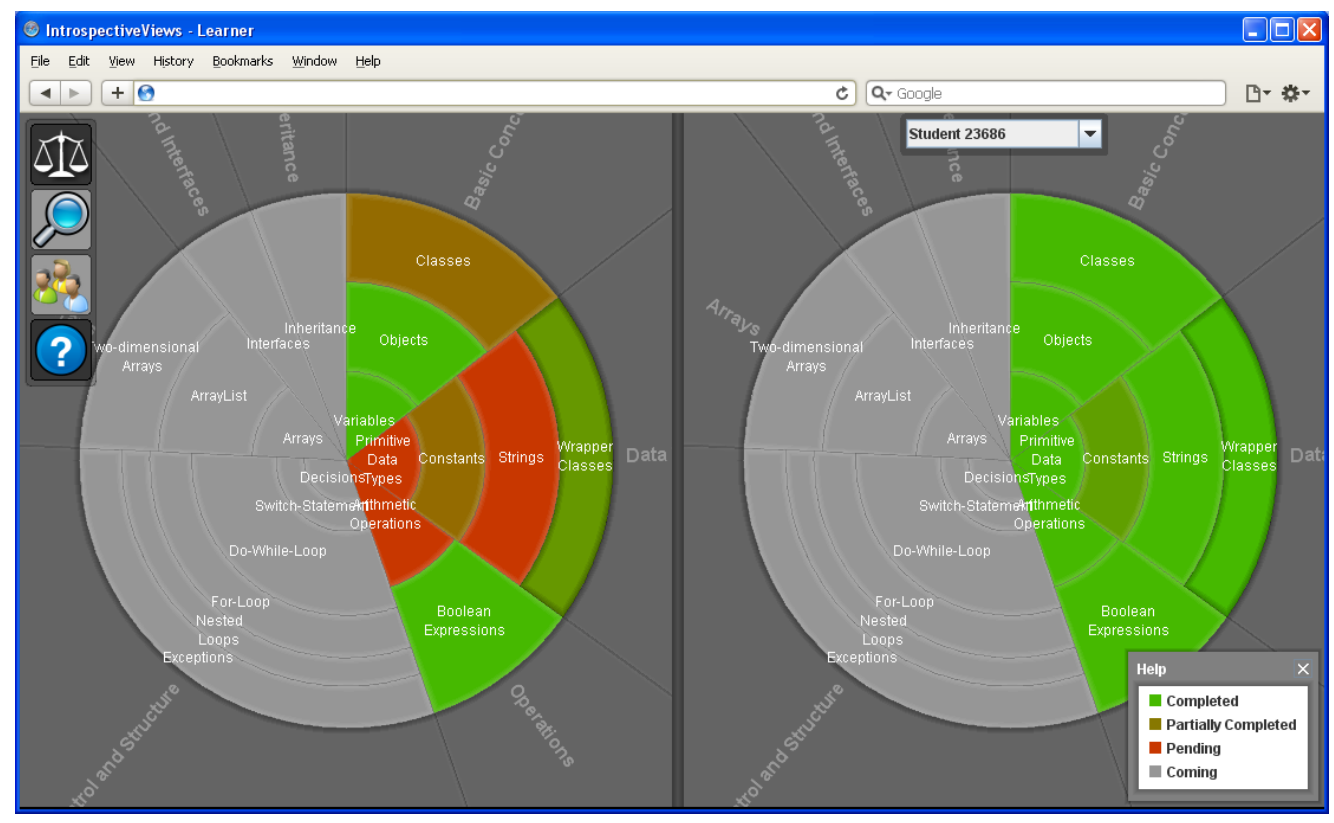

Figure 1. Parallel IntrospectiveViews (predecessor of Progressor). Left pane visualization of the student's own progress; right pane - visualization of a peer's progress. The circular sectors represent the lectures and the annular sectors represent the topics of individual lectures. The shades of the sectors indicate whether the topic has been covered and for the covered ones, denote the progress the student has made. Color screenshots available at: http://www.minervaportals.de/research/introspective-views/.

The visualization (Fig.1) consists of two panes: the left pane displays the student's own model, whereas the right one displays someone else's model. The user can select a peer for comparison from the drop-down menu located on the top of the right pane. Each pane visualizes the respective student's progress as a pie chart consisting of circular sectors representing the class lectures. The lectures are displayed in a clockwise order denoting their presentation sequence, i.e., the order they are taught in class. Lectures may consist of one or more topics, which are represented as annular sectors placed within the circular sector of the corresponding lecture. The color of each annular sector denotes whether the topic has been covered by the teacher in class and, 
for the covered ones, indicates the progress the student has made with respect to the topic. The sectors painted light grey represent the topics that have not yet been covered in class, whereas the sectors painted a shade in the red to green color range represent the sectors that have been covered to date. For the covered topics, the open student model interface visually displays the progress of student knowledge of the topic. Since correct answer to each distinct question contributes to the student overall knowledge of the topic, the topic progress is calculated as the ratio of successfully completed questions to the total question count in the topic. If the ratio equals 0 , i.e., no question has been successfully solved, the sector is painted red. If it equals 1 , i.e., all questions have been completed, the sector appears green. The shades in the range between red and green denote partial completion of the questions.

A very important feature of the Progressor interface is that it integrates open student modeling and access to educational resources. This integration turns a traditional open student model into an adaptive navigation support interface. By clicking on a sector, the interface will display the contents of the corresponding topic, i.e., the list of questions related to the topic (Fig. 2). For each question, the interface provides a visual cue indicating the student's progress and displays the total number of attempts the student has made to complete the quiz and the number of successful attempts. Clicking on a quiz label, will open the quiz in a separate window. 


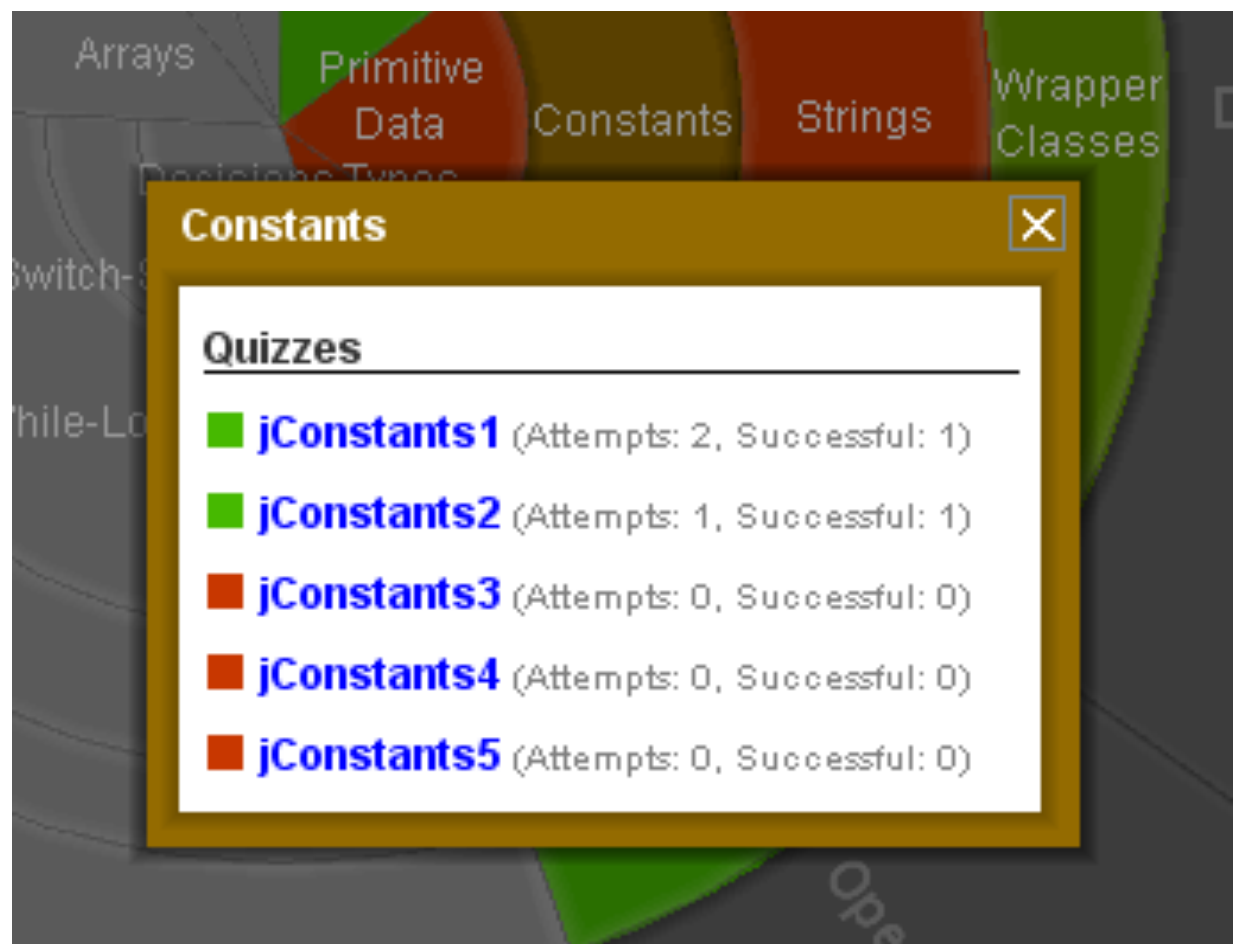

Figure 2. Parallel IntrospectiveViews. Questions on the selected topic.

As our previous study (Hsiao et al., 2011) reveals, Parallel IntrospectiveViews not only helped students to understand the organization of class lectures and accessing questions, but also caused a $28 \%$ increase in the number of attempts to answer each question. However, we believed that the motivational effects could be even stronger, if students were provided with a ranking of their peers' progress. To prove this hypothesis, we developed Progressor, which is an extension of the original Parallel IntrospectiveViews. In this new version (Fig. $3 \& 4$ ), the user is provided with a sortable list of thumbnails of pie charts representing the progress of the user's peers. Unlike the list of student names in the previous version, the list with progress thumbnails provides an immediate overview of the peers' performance. To further increase the value of this list, Progressor allows students to sort the list of peers by name and overall progress (calculated as a sum of progress levels of all topics). By default, the interface displays the models from the most to the least progress. Also, it highlights the models of the three students with the most progress on the top. We believe that displaying the progress of top students in such a manner will make the rest of the class 
more eager to catch up to them. The sorted list contains a thumbnail of the user's own progress, which permits him to determine his/her ranking in the class with respect to either the overall progress or the progress on a selected topic. Also, the list contains a thumbnail with the average progress of the entire class. We believe that such a visualization of the student's own progress compared to the progress of other individual students and the class on average can be a strong motivating factor for completing questions in a timely manner and achieving better scores.

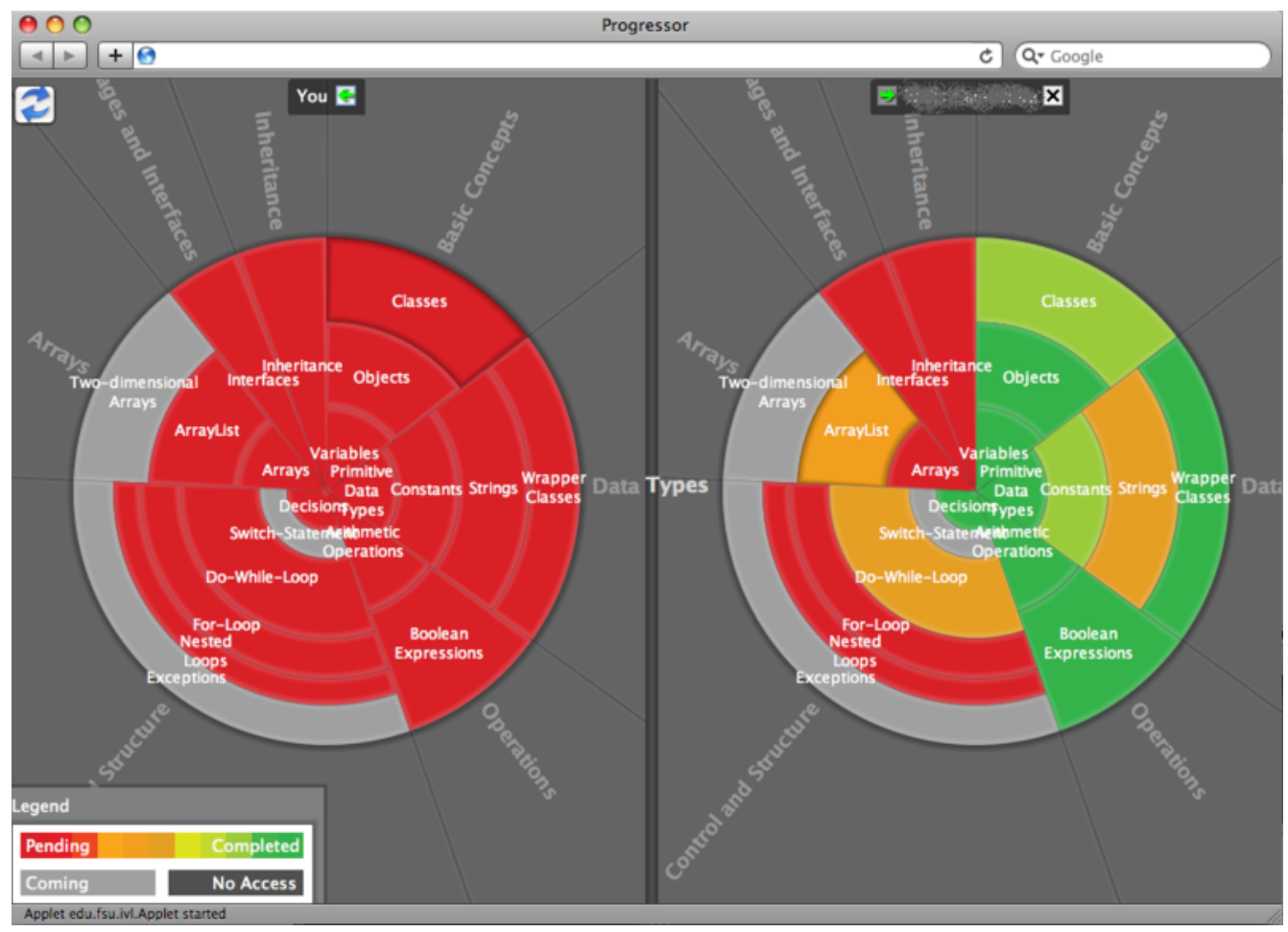

Figure 3. Progressor: Peers' model comparison.

In addition to the preview of peers' progress shown as thumbnails, the user can obtain a detailed view of an individual peer's progress. By clicking on the thumbnail of a particular student, the interface will transform into the one-to-one comparison mode (Fig. 3). In this mode, the user can obtain detailed information about the peer's progress, including the information about his/her progress on individual questions. The detailed pairwise comparison is the key component the Progressor's social navigation mechanism. Examining topic-level the progress of individual peers and the class a 
whole, the student can distinguish relatively easy topics completed by the majority of the students from newer emerging topics that were completed only by a few best students and from advanced topics that were not yet attempted successfully by anyone. This social navigation is critical for selecting the most appropriate topic to work with and the most appropriate question within a topic.

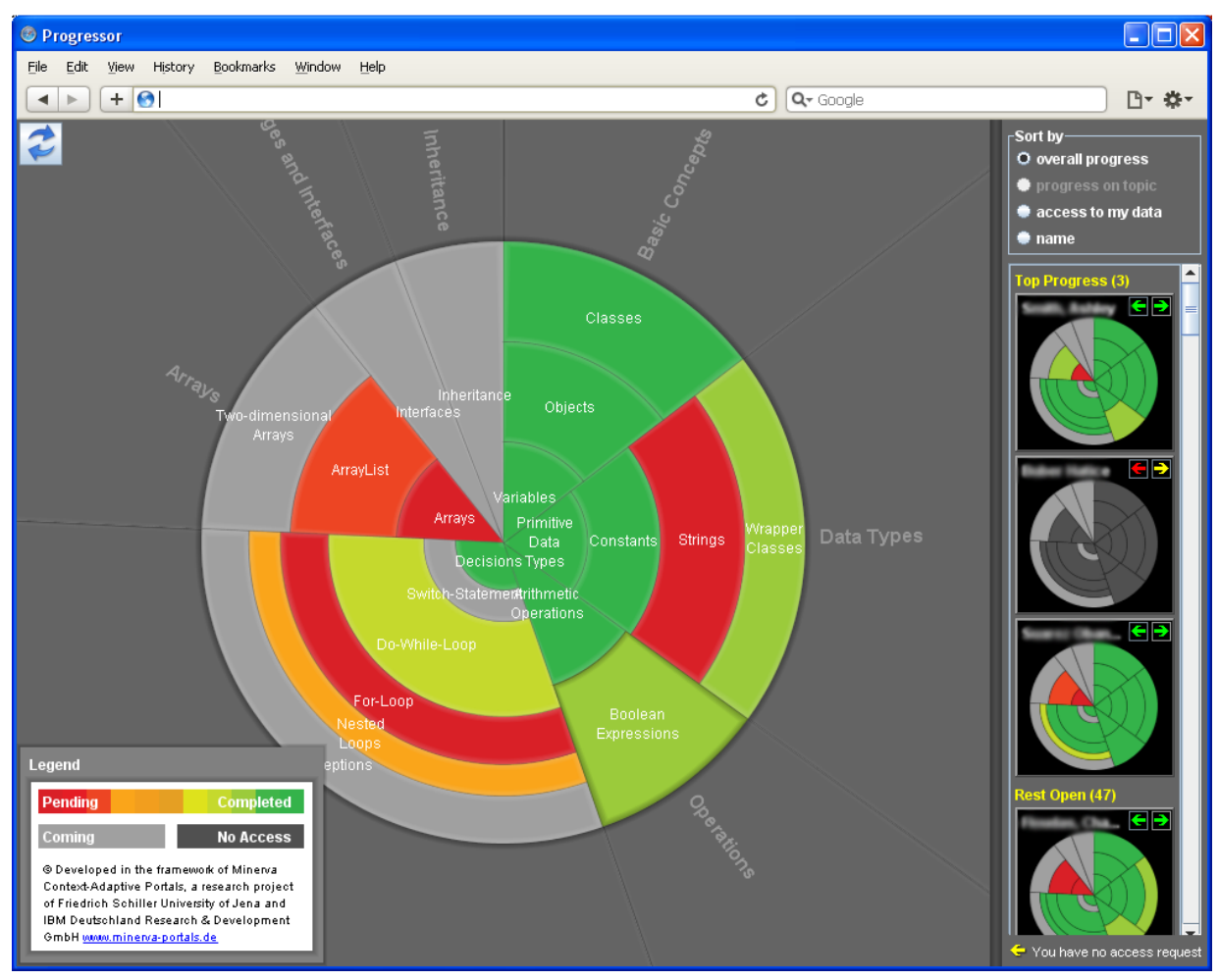

Figure 4. Progressor: Peers' progress is displayed as thumbnails and listed at the side of the user's own model.

To compensate for the increased visibility of peer students' progress, Progressor adds privacy management functionality. The user can grant or revoke access to his/her progress data for each peer individually. The pie charts of the closed models are shaded in dark grey. The interface allows users to submit requests for access to models of other peers. The privacy settings for each peer are displayed on the peer's thumbnail as two arrows: the left arrow indicates the peer's access to the user's data and the right arrow indicates the user's access to the peer's data. The arrows may be in one of the three 
shades: green - access granted, red - no access, yellow - access requested. By clicking on an arrow, the user can change the access status for each peer individually; for example, by clicking on the left arrow the user can grant or revoke the peer's access to his own model. In such a way, the user is able to quickly define the desired progress sharing settings.

\section{Evaluation \& Results}

To assess the impact of our technology, we have conducted a thorough evaluation in a semester-long classroom study. The study was performed in an undergraduate ObjectOriented programming course offered by the School of Information Sciences, University of Pittsburgh, in the Spring 2011 semester. Progressor was introduced to the class at the beginning of the course and served as a voluntary course tool over the entire semester. All student activity with the system was recorded. For every student's attempt to answer a question, the system stored a timestamp, the user's name, the question, quiz, and session IDs, and the correctness of the answer. We also recorded the work with the student model (accessing, comparing and sorting). Pre- and post- tests were administered at the beginning and the end of the semester to measure the students' gains in learning. At the end of the semester, the students were asked to provide their subjective feedback about the system and its features by completing the evaluation questionnaire. To obtain a deeper understanding of how students interact with Progressor, we compared the student work with self-assessment questions through Progressor (Column 3 in Tab. 1) with two other comparable classes: a class that accessed questions a traditional course portal with no social visualization (Column 1 in Tab. 1), and a class that accessed self-assessment questions through Parallel IntrospectiveViews (Column 2 in Tab. 1). Based on our previous experiences with open student modeling in JavaGuide (Hsiao et al., 2010) and Parallel IntrospectiveViews 
(Hsiao et al., 2011), we expected that the new implementation of Progressor would not only encourage the students to work more with the system, but also utilize the traces of social navigation support to result in a better learning outcome.

The rest of the evaluation is organized into five sub-sections. In Section 4.1, we report the system usage. In Sections 4.2 and 4.3 we examine the motivational values of the use of Progressor and discuss the results of social navigation support. In Section 4.4, we evaluate the learning effects. Finally, in Section 4.5, we report on the comprehensive subjective evaluation.

Table 1. Systems usage summary

\begin{tabular}{lll}
\hline 1 & 2 & 3
\end{tabular}

QuizJET QuizJET w/ IV Progressor

$\begin{array}{llll}\text { Parameters } & \mathrm{n}=16 & \mathrm{n}=18 & \mathrm{n}=30\end{array}$

\begin{tabular}{ccccc}
\hline & Attempts & $80.81 \pm 22.06$ & $113.05 \pm 15.17$ & $205.73 \pm 40.46$ \\
Average & Success Rate & $42.63 \% \pm 1.99 \%$ & $71.35 \% \pm 3.39 \%$ & $68.39 \% \pm 4.32 \%$ \\
User & Distinct Topics & $7.81 \pm 1.64$ & $9.06 \pm 1.39$ & $11.47 \pm 1.34$ \\
Statistics & Distinct Questions & $33.37 \pm 6.50$ & $36.5 \pm 5.69$ & $52.7 \pm 6.92$ \\
& Sessions & $3.75 \pm 0.53$ & $4.11 \pm 0.70$ & $8.4 \pm 1.39$ \\
\hline \multicolumn{2}{c}{ Pre-test score $(\mathrm{M} \pm$ SE) } & $9.56 \pm 1.29$ & $6.38 \pm 1.12$ & $3.53 \pm 0.56$ \\
Post-test score (M \pm SE) & $17.12 \pm 0.86$ & $13.71 \pm 1.00$ & $14.61 \pm 0.64$ \\
Normalized Knowledge Gain & $0.36 \pm 0.05$ & $0.43 \pm 0.07$ & $0.57 \pm 0.05$ \\
\hline
\end{tabular}

\subsection{System Usage}

Among 51 registered students, 30 students in the class used Progressor on a regular basis during the course period. On average, students made 205.73 attempts and obtained a $68.39 \%$ success rate in answering the self-assessment questions. Students made significantly more attempts on Progressor than in the two other systems described 
earlier, $F_{13}(1,61)=6.957, p<.05, \eta^{2}=.102 ; F_{23}(1,61)=4.174, p<.05, \eta^{2}=.064$. In addition, students achieved a significantly higher success rate than by accessing selfassessment questions through a traditional portal (QuizJET), $F_{13}(1,61)=12.043, p<.01$, $\eta^{2}=.165$. To assess the Course Coverage, a breadth of course exploration through the new interface, we calculated the number of distinct topics attempted by the student as well as the number of distinct questions attempted. We found that students tried 11.47 distinct topics and 52.7 distinct questions on Progressor on average. There were increases of $46.86 \%$ and $57.92 \%$ of topic and question coverage respectively compared to the usage of the interface without social visualization.

In Table 2, we summarize the usage for each social feature implemented in the open social student modeling interfaces. We counted how many times the students compared their progress to the class on average model or any peer models from the class, how many topics and questions were attempted in their peers' models. We found that students examined the progress of their peers more frequently when a clear list of peers along with their progress is visible. Moreover, we found an even larger increase of student access to learning content through peers model rather than students own model. Note that students can examine their own progress as well as the progress of their peers in details by clicking on a topic sector in either personal or peer model and opening a list of questions with indicators showing question-level progress. An attempt to answer a question can be made by entering a question from any model - own model or a model of any peer. This feature was specifically provided in both interfaces to support social guidance, i.e., encouraging students to examine topics and questions that were already successfully completed by their peers and (which provides an indication that these questions are ready to attempt). An increase of topic and question access through peer models is a good indication that not only comparative learning, but also social 
navigations worked better in Progressor. This is exactly what we observed. Students accessed twice as many topics through their peers' models in Progressor than in IntrospectiveViews. There was also a $35.5 \%$ increase in the questions attempted through the peers' models.

It is also interesting to observe that the students compared their progress with the class on average much less frequently in Progressor. It might be an evidence that peer progress is more important to students that the class average progress. However, it might be a simple result of thumbnail availability, which provided an immediate snapshot comparison without detailed examination. In either case it shows that the students are more eager to examine peer progress in details rather than the class average.

Table 2. Open social student model interfaces usage summary

\begin{tabular}{cccc} 
& & QuizJET w/ IV & Progressor \\
\cline { 2 - 4 } & Parameters & $\mathrm{n}=18$ & $\mathrm{n}=30$ \\
\hline \multirow{2}{*}{ Average } & Class on Average & $3.33 \pm 0.71$ & $0.22 \pm 0.07$ \\
Comparisons & Topics & $6.83 \pm 2.25$ & $8.78 \pm 1.31$ \\
& Questions & $4.00 \pm 0.79$ & $9.00 \pm 1.39$ \\
& & & $6.33 \pm 1.12$ \\
\hline
\end{tabular}

The use of model sorting feature was considerable, but not as high as we expected. Altogether, the students changed the order of sorting from default over 100 times of a bit more than 3 times at average. While this is an indication that sorting feature was valuable, it also hints that the original sorting by progress was most meaningful to the student. Yet, we noticed that some students changed sorting order more frequently and investigated whether an increased use of sorting had any 
connection to student performance. We found that there was significant positive correlation between the frequency of peer model sorting and question attempts and success rate, $r=0.75, p<.01 ; r=0.76, p<.01$. While this result might be interpreted as an impact of sorting on student performance, we believe that it can be better explained by the competitiveness of the students. The more that they worked on the questions and the more that they succeeded in answering the questions, the more the students cared about where they were ranked in the class.

\subsection{Motivational Effects: Confirming the Value of Open Social Student}

\section{Modeling}

In our pre-study (Hsiao et al., 2011), we found that the combination of knowledgebased and social navigation support guided the students to more success in answering the questions. However, the results also showed that the interface actually mediated the motivating effects of progress visualization, making the whole class a bit more cautious than they were without social navigation support. Therefore, the new design of social visualization - Progressor - was intended to enhance the motivational values of Open Social Student Modeling. To evaluate the enhancement, we looked at the differences in the system usage. The major visual improvement from the previous design is the addition of the thumbnail previews of peers' models to access peer student models rather than the original dropdown box with the list of names. Although both interfaces facilitated detailed models comparisons, the dropdown box design didn't display the success of the selected peer, just their name. In contrast, the thumbnails in Progressor provide the users with a straightforward way to navigate through their peers' models, which we assume to yield a more competitive and motivating environment as it reveals the rankings of the student compared to the entire class. The system usage data showed that the students achieved significantly higher success rates with Progressor than with 
the baseline system that has no navigation support at all, $F_{13}(1,61)=12.043, p<.01$, $\eta^{2}=.165$. Such results were consistent with the previous study in demonstrating that the knowledge-based and social navigation support together do a better job in guiding the students to questions that they are ready to handle than knowledge-based navigation support alone. However, in Progressor, students attempted significantly more (with a fairly large variance) of the self-assessment questions than in the QuizJET / course portal combination which had no visualization $\left(F_{13}(1,61)=8.805, p<.01, \eta^{2}=.117\right)$. There are two possible reasons for this sizable variance. Either the students were highly motivated and competitive enough to do more work or some of the students hated the system and made limited use of it. To investigate this issue, we looked at two other parameters such as the average number of distinct questions solved per student and the average amount of time spent on the system (Table 1). We found that students explored more topics and tried a significantly higher number of distinct questions. In addition, the amount of time spent on the system (in terms of the sessions) was doubled. These numbers demonstrated that the students were more engaged with Progressor than the other systems. The average amount of time spent on the system was long enough that we ruled out the possibility that students disliking the system caused the variance. This conclusion can be further supported by the subjective evaluation outlined in Section 4.5.

\subsection{The Evidence of Social Navigation Support}

In our previous work (Brusilovsky et al., 2011; Hsiao et al., 2010), we found evidence that in educational hypermedia with social navigation support, stronger students who exhibit a better understanding of the subject lead weaker students to the most relevant resources by creating navigation trails. Thus, to explore the impact of social features provided by IntrospectiveViews (Hsiao et al., 2011), we also performed a deeper analysis of student activities to examine the same phenomena in Progressor. By taking 
into account the lecture coverage associated with all students' actions, Figure 5 visualizes more than 6100 transactions of all the question attempts performed through Progressor. To distinguish the activities of the Top 3 student models from the rest of class, we color coded the activities into orange and blue. Orange represents the activities generated by the Top 3 students and blue represents the activities of the rest of the students. The semester timeline of the action is marked as the $\mathrm{X}$ axis and the question complexity marked on the $\mathrm{Y}$ axis progresses from easy to complex. We found four interesting zones within this figure. Zone "A" contains the current activity that students performed during the course; it shows that students had been working with the system very consistently throughout the course schedule moving to more and more complex questions as the course progressed from simple to complex topics. Zone "B" represents the timeframe of preparing for the final exam, at which point all ranges of complexities of questions were attempted. Note that the weaker students made most of the attempts in this zone. Zone " $\mathrm{C}$ " contains all of the attempts in which students explored questions that were simpler then those addressed by the current lectures. These attempts were dome predominantly by weaker students, likely to bridge the gaps in acquired knowledge. Zone " $D$ " contains those attempts, which students performed ahead of the course schedule. Surprisingly, this zone actually included a substantial proportion of the attempts. It demonstrated that the system was actually inviting students to challenge themselves to move a little bit ahead of the course pace. It resolved our concerns about the previous design and transformed a conservative environment to a more adventurous one. In addition, we found that the Top 3 students were leading the exploration of future topics. Such an effect was consistent as the course became more complex.

To further examine the social navigation support effect, we categorized the students into two groups based on their pre-test scores (ranging from a minimum of 0 to 
a maximum of 20). As the pre-test scores were positively skewed, we split the two groups by setting the threshold at a score of 7 . Strong students scored 7 points or more (7 13) while weak students scored less than $7(0 \sim 6)$. We found that the strong students generally explored the questions in advance of the weak ones. The effect was especially noticeable in the social navigation adventure zone (Figure 6). Strong students worked on Progressor first and left strong implicit traces for weak students to follow. All zones maintain the same patterns in Figure 5.

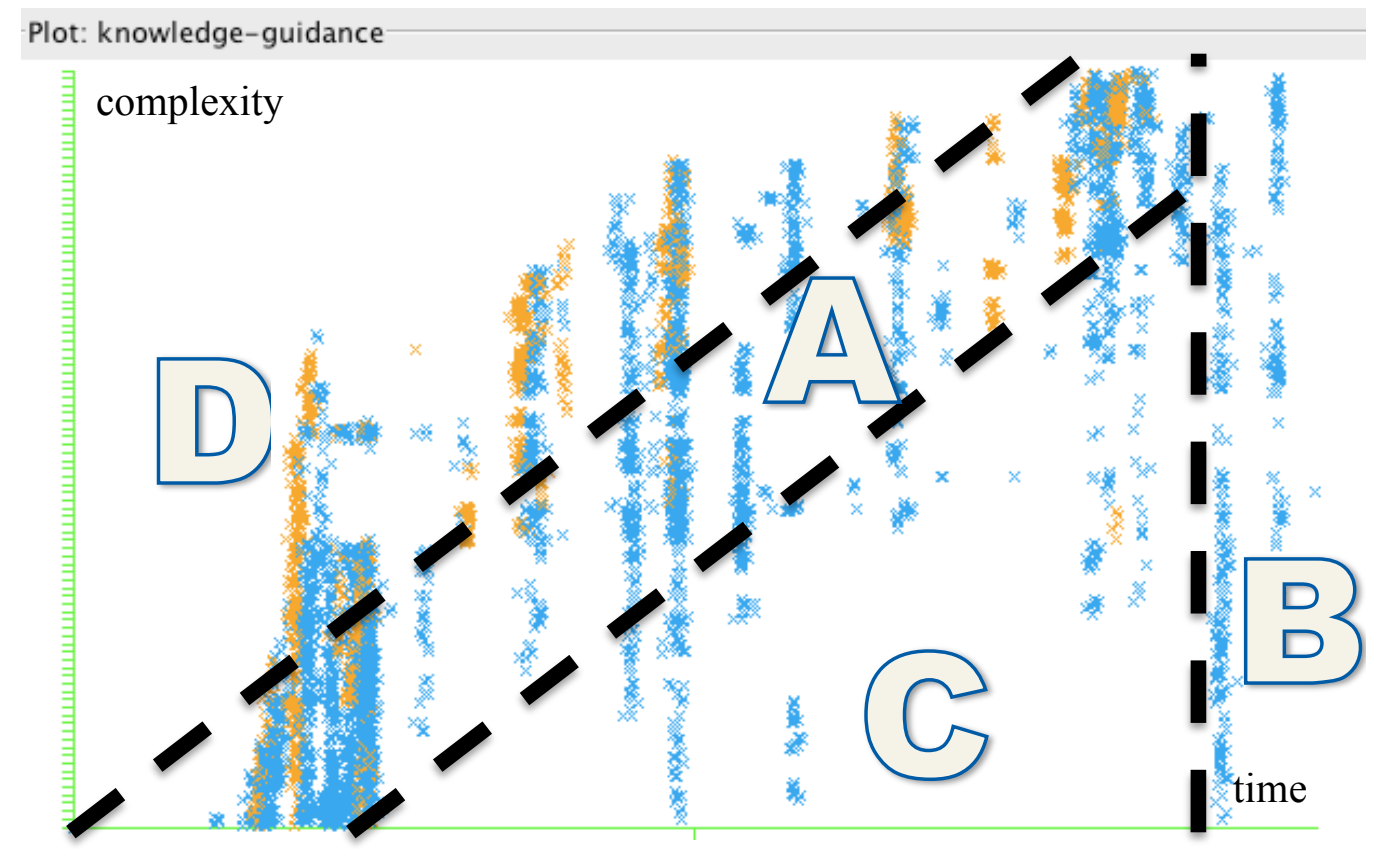

Figure 5. Time distribution of all attempts performed by the students through Progressor. $\mathrm{X}$ axis is the Time; $\mathrm{Y}$ axis is the complexity of the questions. Orange dots represent the Top3 students' actions; blue ones denote the actions belonging to the rest of the class. Zone "A" - lecture stream, zone "B" - final exam period, zone "C" - self-motivated work with the material from earlier lectures, and zone "D" - social navigation adventure. 


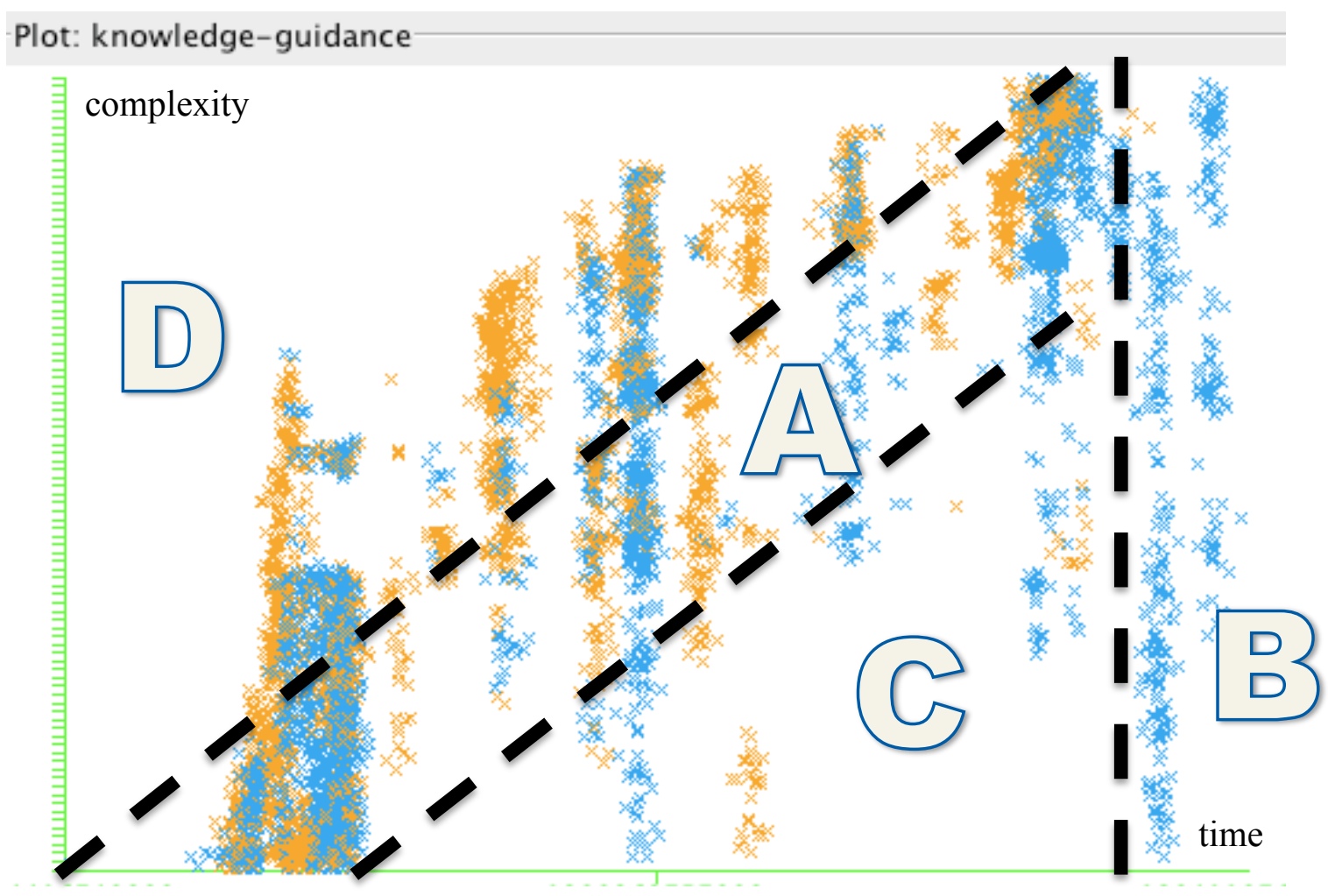

Figure 6. Time distribution of all attempts performed by the students through

Progressor color coded by strong (orange) and weak (blue) knowledge levels.

\subsection{The Effects on Student Learning}

To evaluate the impacts of the open student modeling interface on student learning, we measured the differences between pre- and post- tests. We found that in all systems (QuizJET, IV and Progressor), the students achieved a significant growth in knowledge as measured by pre- and post- test scores, $\mathrm{t}_{1}(15)=6.108, p<.01, \mathrm{t}_{2}(17)=7.203, p<.01$, $t_{3}(29)=14.053, p<.01$. Unfortunately, our assumption of homogeneity of variance was not met, Brown-Forsythe $F(2,61)=12.95, p<.05$. Due to the fact that the group which used Porgressor was particularly weak, it potentially had more room to improve. Therefore, in order to have a fair comparison across groups, we calculated the Normalized Knowledge Gain based on Formula (1). A one-way between-subjects analysis of variance was performed on the normalized knowledge gain as a function of three different interfaces (QuizJET, QuizJET w/ IV, and Progressor). In this case, the assumption of homogeneity of variance was met, Brown-Forsythe $F(2,61)=3.126$, 
$p>.05$. The assumption of normality was met for all systems except QuizJET w/ IV

(Table 3). All other assumptions were met. We found that students obtained significantly higher normalized knowledge gain by working on the self-assessment questions through Progressor $(M=0.572, S E=0.050)$ than QuizJET $(M=0.361, S E=$ $0.050), F(1,61)=1.263, p<.05, \eta^{2}=.021$. It should be noted that all three studies were performed in a non-controlled classroom context, where the systems were used as supplementary course tools. The students were able to learn the subject through many other methods in addition to the self-assessment questions from the explored systems.

$$
N K G=\frac{\text { posttest }- \text { pretest }}{\max \text { score }- \text { pretest }}
$$

Table 3. Test of normality of normalized knowledge gain for each system interface.

\begin{tabular}{lccc}
\hline System & Shapiro-Wilk $W$ & $d f$ & $p$ \\
QuizJET & 0.936 & 16 & 0.302 \\
QuizJET w/ IV & 0.873 & 18 & 0.020 \\
Progressor & 0.958 & 30 & 0.275 \\
\hline
\end{tabular}

\subsection{Subjective Evaluation}

To examine the students' attitudes toward Progressor, we asked students to complete evaluation questionnaires at the end of the semester. There were 31 students who completed the questionnaire, 17 male and 14 female, however, we excluded the answers of one 1 student who completed the questionnaire but have not used the system. In the survey, there were 22 questions, including several that addressed the usability of interface elements to improve users' satisfaction with the interface in general. Users were asked to evaluate the questions on a 5-point Likert scale and to provide free-form comments as they wished. We divided the 22 questions into 5 categories, including 
Usefulness, Ease of Use, Ease of Learning, Satisfaction and Privacy \& Data Sharing (Table 4). We found that male users had more positive attitudes toward Progressor than females did across all categories, except for the Ease of Learning aspect (not significant) as reported in Figure 6. On average, we did not see significant differences between the genders with regard to the system. However, if we zoomed in to examine the feedback by question, we found that male students held significantly more positive attitudes than female students in two aspects: male students felt that the interface helped them to better plan for the class work and they thought the comparison mode of the interface helped them to find the classmates who could help them with difficult topics $\left(F\left(1,29^{1}\right)=16.588, p<.01 ; F(1,30)=4.598, p<.05\right)$.

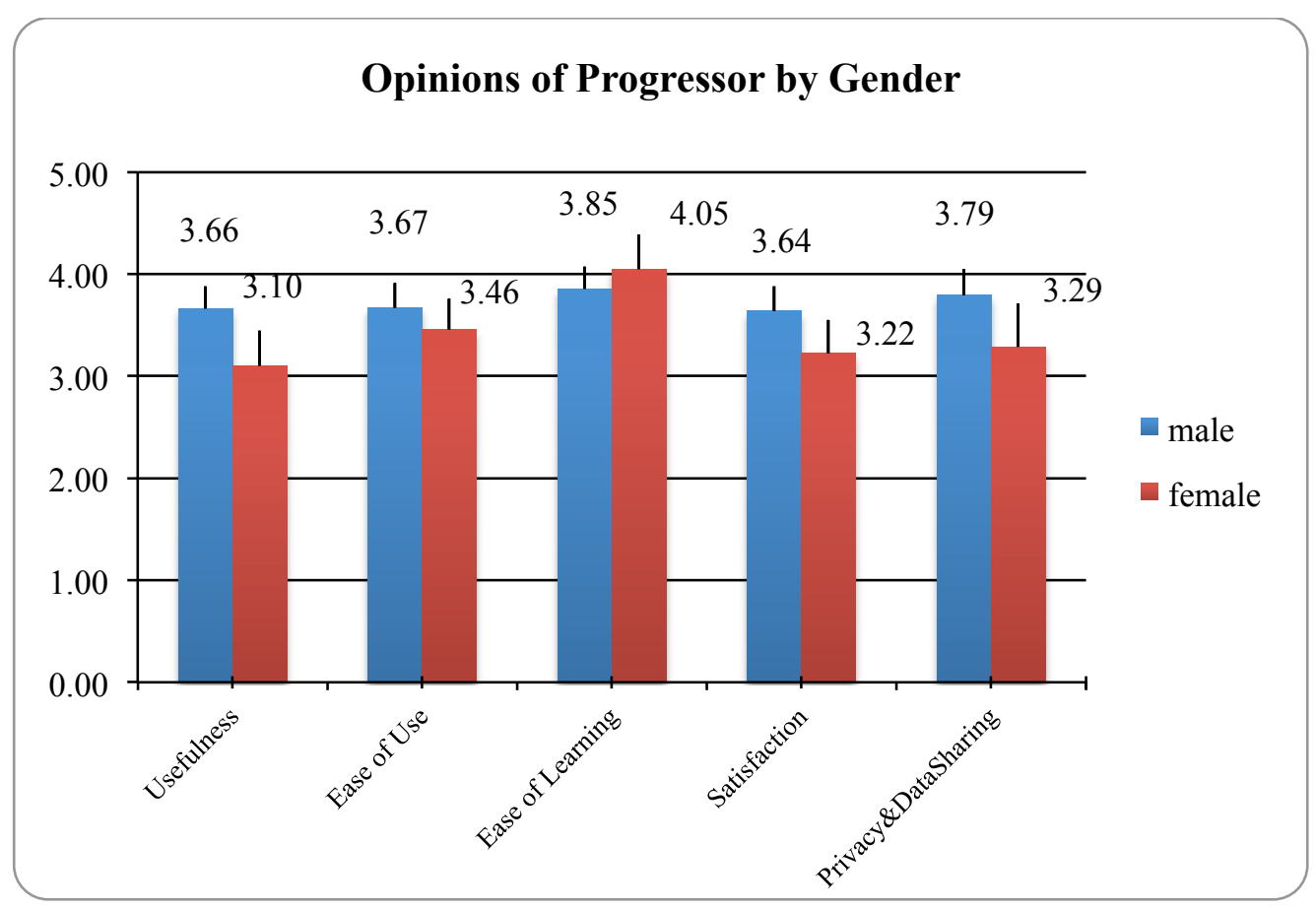

\section{Figure 7. Student opinions about Progressor by gender}

The distribution of student answers is presented in Figure 8. In the Usefulness

\footnotetext{
${ }^{1}$ One of the survey participants did not answer this question (A.3). The percentile was calculated based on the responses from the remainder of the participants.
} 
category, $74.2 \%$ of the students strongly agreed or agreed that the open social student modeling interface helped them to understand how the class content was organized. $74.2 \%$ of the students agreed or strongly agreed that the interface helped them to identify their weak points. For the question about whether or not the interface helped students to plan their class work, students had differing responses; however, $50 \%$ of them held no strong opinion. As the system was not required for the class, we can understand why the students did not have strong feelings of either agreement or disagreement. $51.6 \%$ of the students thought the interface really helped them to access the questions. $54.8 \%$ of them strongly agreed or agreed the thumbnails view motivated them to progress in the questions. $51.6 \%$ of the students agreed that the comparison mode motivated them to progress with the questions.

Considering the Ease of Use \& Ease of Learning in the system, students found it easy to remember how to use it $(83.8 \%)$ and learned how to use it quickly $(77.4 \%)$ They felt that the interface was easy to use (70.9\%), user-friendly $(70.0 \%)$ and easy to learn (70.9\%). $83.4 \%$ of students agreed or strongly agreed that the interface required fewer steps to accomplish the task. There was only $3.33 \%$ strong disagreement across all the questions of this category. In the category of Satisfaction, $61.3 \%$ were strongly satisfied with the system and $61.3 \%$ of them would recommend it to their classmates. They generally felt that the interface was fun $(61.7 \%)$ and pleasant $(87.1 \%)$ to use. In terms of Privacy and Data Sharing perspectives, most of the students had positive attitudes in this category of questions. They had roughly the same proportion of agreement (ranging from neutral to strongly agree) on displaying their models anonymously and generally appreciated the feature of access by request. $54.8 \%$ of the students appreciated the feature of comparing their progress with others. $61.3 \%$ of them felt comfortable in sharing their progress with others. Figure 8 shows the detailed percentages for each 
question.

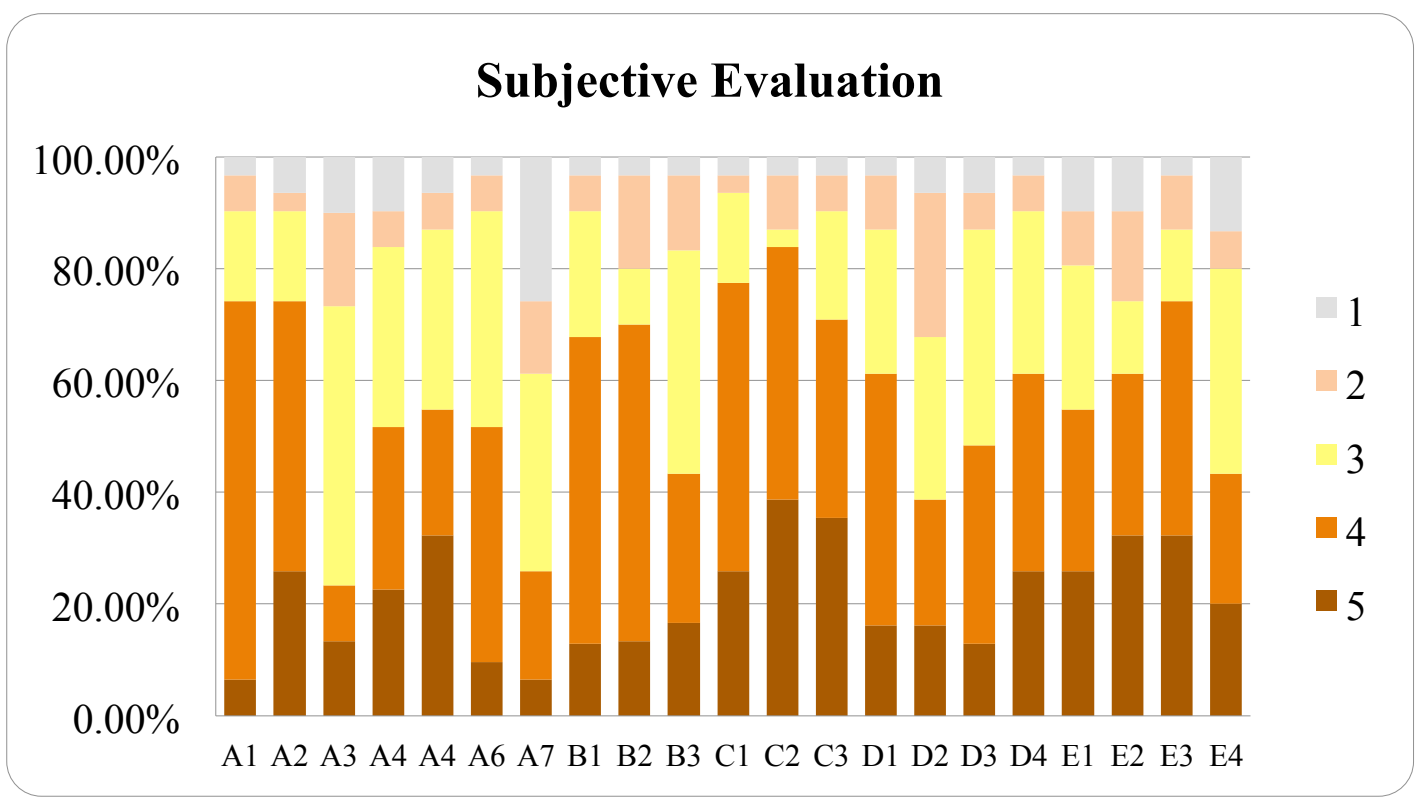

Figure 8. Summary of subjective evaluation on Progressor . The answers ranged from strongly agree (5) to strongly disagree (1)

Table 4. Survey questions for Progressor

Usefulness

A.1. The interface helps me to understand how the class content is organized.

A.2. The interface helps me to identify my weak points.

A.3. The interface helps me to plan my class work.

A.4. The interface helps me to access questions.

A.5. The thumbnails view motivates me to progress on the questions.

A.6. The comparison mode of the interface motivates me to progress on the questions.

A.7. The comparison mode of the interface helps me to find the classmates who can help on difficult topics.

\section{Ease of Use}

B.1. The interface is easy to use.

B.2. The interface is user-friendly.

B.3. The interface requires the fewest steps possible to accomplish what I want to do.

\section{Ease of Learning}

C.1. I learned how to use the interface quickly. 
C.2. I easily remember how to use the interface.

C.3. It is easy to learn how to use the interface.

\section{Satisfaction}

D.1. I am satisfied with the interface.

D.2. The interface is fun to use.

D.3. The interface is pleasant to use.

D.4. I would recommend the interface to my classmates.

\section{Privacy and Data Sharing}

E.1. I like the idea of comparing my progress with other students.

E.2. I feel comfortable sharing my progress with others.

E.3. I do not mind that my progress is displayed anonymously in the average progress of the entire class.

E.4. I like the access by requests feature.

E.5. I would like to view progress of another student because:

1. S/He and I are friends

2. I know s/he is a good student

3. I know s/he is good at specific topic

4. I am just curious

Other

\section{SUMMARY \& FUTURE WORK}

This paper described an innovative Web-based interface, Progressor, which was designed to help students to find the most relevant resources in a large collection of parameterized self-assessment questions about programming. The interface was built based on the open social student model. Students were able to navigate through all of their peers' models and to compare them to one another. A semester-long study comparing the student work with the new tool against data collected during earlier semesters was conducted. We found that students used the systems heavily in spite of the fact that their use was not mandatory. We also 
confirmed the motivational value of personalized social navigation support provided by Progressor. The results revealed that the interface encouraged students to explore more topics and motivated them to work ahead of the course schedule. A deeper analysis of the social navigation support mechanism revealed that the top students, who provided implicit social navigation support for the rest of the class, successfully led the way for weaker students to discover the most relevant resources by creating pathways. In addition, we also found that there were significant positive correlations between the frequencies of peer model sorting and question attempts and success rate. The more they worked on the questions and the more often they succeeded in answering the questions, the more students cared about where they were ranked in the class. The study results also demonstrated that students were more engaged with the system by spending more time in working with self-assessment questions, attempting more questions and achieving higher success rates in answering them. Last but not least, students praised the interface and indicated that they would recommend it to other students. Moreover, males appeared to be more favorable towards the interface in general. Significant differences between males and females were found in their opinions regarding the usefulness of the interface.

In the future, we would like to extend the current approach to a larger scale. While the results in this study were encouraging, we believe that the current approach has not yet reached its full potential. For example, even though students were able to discover more topics and questions by following the implicit trails from the stronger students, there are still a reasonable number of unexplored questions. According to our prior work, providing adaptive navigation support significantly increases the quality of student learning and student motivation to 
work with non-mandatory learning content. We plan to have a richer integration

of open social student modeling with adaptive navigation support. Furthermore,

we seek to investigate more deeply privacy and data sharing in open social student

modeling interfaces.

\section{ACKNOWLEDGMENTS}

This research is partially supported by the National Science Foundation under Grants

0447083 and 0840597 . The development of Progressor was partially carried out in the

Minerva Context-Adaptive Portals project funded by the IBM Deutschland Research \&

Development GmbH.

\section{REFERENCES}

F. Bakalov, B. König-Ries, A. Nauerz and M. Welsch, 2010. IntrospectiveViews: An Interface for Scrutinizing Semantic User Models. In: P. De Bra, A. Kobsa and D. Chin, eds., 18th International Conference on User Modeling, Adaptation, and Personalization (UMAP 2009) Lecture Notes in Computer Science 6075, Springer, Big Island, HI, USA, 219-230.

L. Barolli, A. Koyama, A. Durresi and G. De Marco, 2006. A web-based e-learning system for increasing study efficiency by stimulating learner's motivation. Information Systems Frontiers, 8 (4), 297-306.

P. Brusilovsky, 2007. Adaptive navigation support. In: P. Brusilovsky, A. Kobsa and W. Neidl, eds. Adaptive navigation support. Lecture Notes in Computer Science 4321. Berlin Heidelberg New York: Springer-Verlag,263-290.

P. Brusilovsky, 2008. Social Information Access: The Other Side of the Social Web. In: V. Geffert, et al., eds., SOFSEM 2008, 34th International Conference on Current Trends in Theory and Practice of Computer Science Lecture Notes in Computer Science 4910, Springer Verlag, High Tatras, Slovakia, 5-22.

P. Brusilovsky, G. Chavan and R. Farzan, 2004. Social adaptive navigation support for open corpus electronic textbooks. In: P. De Bra and W. Nejdl, eds., Third International Conference on Adaptive Hypermedia and Adaptive Web-Based Systems (AH'2004) Lecture Notes in Computer Science 3137, Springer-Verlag, Eindhoven, the Netherlands, 24-33.

P. Brusilovsky, I.H. Hsiao and Y. Folajimi, 2011. QuizMap: Open Social Student Modeling and Adaptive Navigation Support with TreeMaps. In: C. Kloos, D. Gillet, R. Crespo García, F. Wild and M. Wolpers, eds. QuizMap: Open Social Student Modeling and Adaptive Navigation Support with TreeMaps. Lecture Notes in Computer Science 6964. Berlin / Heidelberg: Springer 71-82.

S. Bull, 2004. Supporting learning with open learner models. 4th Hellenic Conference on Information and Communication Technologies in Education A, Athens, Greece, 47-61. 
S. Bull and J. Kay, 2007. Student Models that Invite the Learner In: The SMILI:) Open Learner Modelling Framework. International Journal of Artificial Intelligence in Education, 17 (Volume 17, Number 2 / 2007), 89-120.

Z.-H. Chen, C.-Y. Chou, Y.-C. Deng and T.-W. Chan, 2007. Active Open Learner Models as Animal Companions: Motivating Children to Learn through Interacting with My-Pet and Our-Pet. International Journal of Artificial Intelligence in Education, 17 (Volume 17, Number 2 / 2007), 145-167.

A.T. Corbett and J.R. Anderson, 1995. Knowledge tracing: Modelling the acquisition of procedural knowledge. User Modeling and User-Adapted Interaction, 4 (4), 253-278.

A. Dieberger, 1997. Supporting social navigation on the World Wide Web. International Journal of Human-Computer Interaction, 46, 805-825.

A. Dieberger, P. Dourish, K. Höök, P. Resnick and A. Wexelblat, 2000. Social navigation: Techniques for building more usable systems. interactions, 7 (6), 3645.

V. Dimitrova, 2003. STyLE-OLM: Interactive Open Learner Modelling. Int. J. Artif. Intell. Ed., 13 (1), 35-78.

V. Dimitrova, J. Self and P. Brna, 2001. Applying interactive open learner models to learning technical terminology. In: M. Bauer, P.J. Gmytrasiewicz and J. Vassileva, eds., 8th International Conference on User Modeling, UM 2001 Lecture Notes on Artificial Intelligence 2109, Springer-Verlag, Berlin, 148-157.

R. Farzan and P. Brusilovsky, 2006. Social navigation support in a course recommendation system. In: V. Wade, H. Ashman and B. Smyth, eds., 4th International Conference on Adaptive Hypermedia and Adaptive Web-Based Systems (AH'2006) Lecture Notes in Computer Science 4018, Springer Verlag, Dublin, Ireland, 91-100.

R. Farzan and P. Brusilovsky, 2008. AnnotatEd: A social navigation and annotation service for web-based educational resources. New Review in Hypermedia and Multimedia, 14 (1), 3-32.

R. Farzan and P. Brusilovsky, 2008. Where did the Researchers Go? Supporting Social Navigation at a Large Academic Conference. The 19th ACM Conference on Hypertext \& Hypermedia, Pittsburgh, Pennsylvania, USA, 203-211.

I.-H. Hsiao, F. Bakalov, P. Brusilovsky and B. König-Ries, 2011. Open Social Student Modeling: Visualizing Student Models with Parallel Introspective Views. 19th International Conference on User Modeling, Adaptation, and Personalization, UMAP 2011 Springer-Verlag, Girona, Spain, 171-182.

I.-H. Hsiao, F. Bakalov, P. Brusilovsky and B. König-Ries, 2011. Open Social Student Modeling: Visualizing Student Models with Parallel IntrospectiveViews. UMAP Volume 6787/2011, Lecture Notes in Computer Science, Girona, Spain, 171182.

I.-H. Hsiao, P. Brusilovsky and S. Sosnovsky, 2008. Web-based Parameterized Questions for Object-Oriented Programming. World Conference on E-Learning, E-Learn 2008, Las Vegas, USA, 3728-3735.

I.-H. Hsiao, S. Sosnovsky and P. Brusilovsky, 2010. Guiding students to the right questions: adaptive navigation support in an E-Learning system for Java programming. Journal of Computer Assisted Learning, 26 (4), 270-283.

J. Janssen, C. Tattersall, W. Waterink, B. van den Berg, R. van Es, C. Bolman and R. Koper, 2007. Self-organising navigational support in lifelong learning: How predecessors can lead the way. Computers and Education, 49, 781-793. 
A. Kavcic, 2004. Fuzzy User Modeling for Adaptation in Educational Hypermedia. IEEE Transactions on Systems, Man, and Cybernetics, 34 (4), 439-449.

J. Kay, 2008. Lifelong Learner Modeling for Lifelong Personalized Pervasive Learning. IEEE Transactions on Learning Technologies, 1 (4), 215-228.

J. Kurhila, M. Miettinen, P. Nokelainen and H. Tirri, 2002. EDUCO - A collaborative learning environment based on social navigation. In: P. De Bra, P. Brusilovsky and R. Conejo, eds., Second International Conference on Adaptive Hypermedia and Adaptive Web-Based Systems (AH'2002) Lecture Notes in Computer Science 2347, Málaga, Spain, 242-252.

A. Mabbott and S. Bull, 2004. Alternative Views on Knowledge: Presentation of Open Learner Models. In: J.C. Lester, R.M. Vicari and F. Paraguassu, eds. Alternative Views on Knowledge: Presentation of Open Learner Models. Lecture Notes in Computer Science 3220. Berlin / Heidelberg: Springer,131150.

J.A. Méndez, C. Lorenzo, L. Acosta, S. Torres and E. González, 2006. A web-based tool for control engineering teaching. Computer Applications in Engineering Education, 14 (3), 178-187.

R. Mertens, R. Farzan and P. Brusilovsky, 2006. Social navigation in web lectures. In: U.K. Wiil, P.J. Nürnberg and J. Rubart, eds., Seventeenth ACM Conference on Hypertext and Hypermedia (Hypertext 2006) ACM Press, Odense, Denmark, 41-44.

A. Mitrovic and B. Martin, 2007. Evaluating the Effect of Open Student Models on Self-Assessment. International Journal of Artificial Intelligence in Education, 17 (2), 121-144.

J. Vassileva, 2008. Toward Social Learning Enviroments. IEEE Transaction on Learning Technologies, 1 (4), 199-214.

J. Vassileva and L. Sun, 2008. Evolving a Social Visualization Design Aimed at Increasing Participation in a Class-Based Online Community. International Journal of Cooperative Information Systems (IJCIS), 17 (4), 443-466.

A. Wexelblat and P. Mayes, 1999. Footprints: History-rich tools for information foraging. ACM Conference on Human-Computer Interaction (CHI'99), Pittsburgh, PA, 270-277.

J.-D. Zapata-Rivera and J.E. Greer, 2000. Inspecting and Visualizing Distributed Bayesian Student Models. INTELLIGENT TUTORING SYSTEMS Lecture Notes in Computer Science 544-553. 